\title{
Acidosis láctica en paciente diabético tratado con metformina
}

\author{
M. SOLANO REMIREZ, C. GONZÁLEZ ARENCIBIA, M. ÁLVAREZ FRÍAS, \\ B. LLORENTE DÍAZ, M. ECHEGARAY AGARA
}

Servicio de Medicina Interna. Hospital de Navarra. Pamplona

\author{
LACTIC ACIDOSIS IN DIABETIC PATIENT TREATED WITH MET- \\ FORMIN
}

\section{RESUMEN}

Presentamos el caso de un varón diabético tipo 2 de reciente diagnóstico en tratamiento con metformina que sufre acidosis metabólica. La metformina (junto a fenformina y la butformina) es antidiabético oral, de la familia de las biguanidas, que constituyen un grupo farmacológico utilizado en el tratamiento de la diabetes mellitus no insulíndependiente. Su principal indicación es la asociación con otros grupos de antidiabéticos orales en el tratamiento de pacientes diabéticos obesos con mal control metabólico, presentando indudables beneficios en este tipo de pacientes. En algunos pacientes su uso puede predisponer a acidosis láctica, especialmente en aquellos que presentan factores predisponentes asociados (insuficiencia respiratoria, insuficiencia hepática o enfermedades cardiovasculares) y/o empleo de dosis elevadas del fármaco. Es por ello que describimos su farmacocinética, indicaciones terapéuticas y su correcto manejo en este tipo de pacientes diabéticos.

PALABRAS CLAVE: Metformina. Biguanidas. Acidosis láctica. Diabetes mellitus. Parada cardiorrespiratoria.

\begin{abstract}
We present a case of metabolic acidosis ina man, recently diagnosed with type 2 Diabetes Mellitus under treatment with metformin. Metformin (along with Fenformin and Butformin) is an oral antihyperglycemic agent belonging to the biguanide group employed in the treatment of non insulin dependent diabetes (NIDDM). Its main use is in associattion with other oral agents in obese diabetic patients with difficult metabolic control. In some of these patients, clearly beneficial developed lactic acidosis, specially in those who have predisposing factors (respiratry failure, liver disease or cardiovascular disease) and/or those who require high dosis. For this reason we describe it's pharmacokinetics, therapeutic indications and its correct use in this type of diabetic patient.
\end{abstract}

KEY WORDS: Metformin. Biguanide. Lactic acidosis. Diabetes mellitus. Cardiorespiratory failure.

Solano Remirez M, González Arencibia C, Álvarez Frías M, Llorente Díaz B, Echegaray Agara MN. Acidosis láctica en paciente diabético tratado con metformina. An Med Interna (Madrid) 2004; 21: 288-290.

\section{INTRODUCCIÓN}

La acidosis láctica es un trastorno ácido-básico consecutivo a la acumulación de ácido láctico. Este se acumula en la célula como contrapartida al ácido pirúvico reducido resultante de la degradación de la glucosa a través del metabolismo anaerobio microsomal que puede culminar en $\mathrm{CO} 2 \mathrm{H} 2 \mathrm{O}$ si sigue la vía del ciclo de Krebs. Cualquier fenómeno que acelere la glucólisis originará la acumulación de piruvato; la disponibilidad de $\mathrm{O} 2$ y una función mitocondrial adecuada favorecerá la utilización de aquel en el ciclo del ácido cítrico mediante la oxidación del NADH a NAD+, pudiendo entrar en la vía de la gluconeogénesis. Si la funcionalidad mitocondrial falla y/o existe dificultad en la disponibilidad del oxígeno, la única posibilidad es la oxidación de piruvato en lactato, con la consiguiente acumulación de éste. La etiología de la acidosis podemos clasificarla en dos grandes grupos, de forma genérica, según se deba a la existencia de hipoxia celular o no sea debida a ésta (en este caso, secundaria a sustancias que la favorezcan y/o errores innatos del metabolismo). Entre los fármacos que asocian acidosis láctica está descritos, en la literatura, las biguanidas $\mathrm{y}$, dentro de estas, la metformina $(1,2)$.

\section{CASO APORTADO}

Se trata de un varón de 58 años de edad. En los antecedentes personales presenta HTA en tratamiento con valsartan (DIOVAN®) 160 $\mathrm{mg} 1 \mathrm{cdo} / 12 \mathrm{~h}$ y verapamilo (MANIDON RETARD®) $240 \mathrm{mg}$ 1cdo/12h; Diabetes Mellitus tipo 2, diagnosticada hacía 2 semanas, en tratamiento con metformina (GLAFONILv) $500 \mathrm{mg} 1 \mathrm{cdo} / 6 \mathrm{~h}$; hiperlipemia en tratamiento con gemfibrozilo (LOPID®) $900 \mathrm{mg}$ $1 \mathrm{cdo} / 24 \mathrm{~h}$. Asintomático los días previos, al levantarse por la mañana y sin causa desencadenante previa, comienza con sensación de mareo y cansancio, por lo que, él mismo, decide tomarse la TA, objetivando cifras de 90/70, y se realiza una glucemia capilar (Dtx: 190). Seguidamente el paciente presenta empeoramiento del cuadro clínico acompañado de importante cortejo vegetativo y no objetivando TA en la exploración, por lo que avisa a SOS que envía una UCI. móvil, que se encuentra con un paciente cianótico y muy afectado, con un ritmo aparentemente sinusal a una frecuencia cardiaca de $28 \mathrm{lpm}$, en el monitor. Se le administra $1,5 \mathrm{mg}$ de atropina ev, siendo trasladado

Trabajo aceptado: 8 de enero de 2004

Correspondencia: Manuel Solano Remirez. Juan Bosco, 17-2 izquierda. 31007 Pamplona (Navarra). 
al Servicio de Urgencias donde se objetiva un cuadro de hipotensión con mala perfusión periférica, taquipneico a $46 \mathrm{rpm}$, ausencia de pulsos periféricos y dolor abdominal generalizado sin focalidad ni signos de irritación peritoneal. En el ECG se observa una frecuencia cardiaca de 381.p.m con elevación generalizada de ondas T de morfología picuda. Bruscamente el paciente entra en parada cardiorrespiratoria (PCR) por disociación electromecánica (DEM). Se realizan maniobras de soporte vital avanzado (SVA) requiriéndose intubación orotraqueal (IOT), masaje cardiaco y se administran $3 \mathrm{mg}$ de adrenalina. La situación hemodinámica es inestable por lo que se inició perfusión con fármacos simpaticomimérgicos. Así mismo se inicia perfusión con bicarbonato $1 \mathrm{M}$ y salbutamol, procediendo a su ingreso en UCI. En dicho Servicio, el paciente se encuentra sedado y conectado a ventilación mecánica. Mantiene constantes vitales estables con tensión arterial (TA) de 130/70, una temperatura de $36^{\circ} \mathrm{C}$ y una frecuencia respiratoria (FR) de $14 \mathrm{rpm}$. El paciente está sedado presentando, a la exploración, pupilas midriáticas, reflejos oculomotores presentes, con buena perfusión periférica y pulsos palpables. En la auscultación cardiaca objetiva bradicardia sin soplos y, en la auscultación pulmonar, murmullo vesicular normal (MVC) con aceptable ventilación de ambos campos pulmonares. El abdomen es globuloso, blando, depresible, no doloroso a palpación y no se palpan masas ni megalias, auscultándose peristaltismo de progresión. La TAC abdominal, ecocardiograma transtorácico y radiografía de tórax son normales. En el ECG se observa un ritmo sinusal a 77 l.p.m con onda T picudas en derivaciones precordiales y discreto descenso de S-T de V1 a V2. La Gasometría arterial, con una FiO2 al 21\% y PEEP de 8, nos da un pH 7,21, pO2 61, pCO2 46, HCO3 18, SatO2 86 y un exceso de base (EAB) de -10. En la analítica de sangre obtenemos 9.330 leucocitos con fórmula normal, glucosa 551, Urea 40, Creatinina 1,9, ASAT 231, ALAT 270, CPK 20, troponina 0,0, K 74 $\mathrm{mmol} / \mathrm{L}$, Na $131 \mathrm{mmol} / \mathrm{L}$ y un anión GAP de 29,3 (Tabla I). En al analítica de orina ausencia de cetosis. Desde el punto de vista evolutivo, a las seis horas de su ingreso en UCI. presenta clara mejoría del estado hemodinámico por lo que se procede a la retirada progresiva de la perfusión de simpaticomiméticos, presentando tendencia a elevación de TA que requirió empleo de urapidilo e.v. Las constantes vitales se mantienen dentro de la normalidad con frecuencia cardiaca entre valores de 75 a 100 l.p.m, recuperación del ritmo sinusal, diuresis mantenida y estable, permaneciendo afebril, por lo que se procedió a la retirada de la ventilación mecánica mediante extubación. El paciente mantiene respiración espontánea con una saturación de $\mathrm{O} 2$ de $126 \%$, con oxigenoterapia en gafas nasales a $4 \mathrm{~L} / \mathrm{m}$. Dada la buena evolución del cuadro clínico se decide su traslado, para control y seguimiento evolutivo, a planta de hospitalización. Durante su estancia en planta el paciente se encuentra asintomático, con situación hemodinámica estable y valores analíticos que evolucionan a la normalidad, resolviéndose el cuadro clínico que motivó su ingreso.

\section{DISCUSIÓN}

El lactato es un producto final de la glicólisis, utilizado por el hígado y el riñón para la gluconeogénesis. En situaciones de aumento de la glicólisis anaerobia, puede haber superproducción de lactato desde el piruvato y fallar los mecanismos reguladores, acumulándose lactato, el cual se disocia rápidamente formando hidrogeniones que son los causantes de la acidosis láctica. Se define lactacidemia cuando encontramos niveles de lactato en plasma de $2-5 \mathrm{mmol} / \mathrm{L}$, caracterizado por acidosis metabólica definida por un $\mathrm{pH}$ menor de 7,35 con bicarbonato plasmático menor de $22 \mathrm{mmol} / \mathrm{L}$. La lacticacidemia ocurre, sin repercusión metabólica ni clínica, en pacientes diabéticos tipo 2 tratados con biguanidas; su progresión a acidosis láctica se produce por asociación con determinadas condiciones adversas (3). Se distinguen dos tipos de acidosis láctica (Tabla II).

Los datos de laboratorio anormales indicadores de acidosis láctica son el anión GAP elevado, exceso de base (ABE) en la gasometría arterial (existe escasa correlación entre el pH arterial y la acidosis láctica) y los niveles plasmáticos de ácido láctico (mayores de $2 \mathrm{mmol} / \mathrm{L}$ ) (4). Los niveles sanguíneos de lactato pueden constituirse como valores pronósticos (5). Una de las causas de acidosis láctica es el empleo de metformina en el tratamiento de la diabetes mellitus tipo $2(6,7)$. Las biguanidas inhiben la gluconeogénesis en el hígado, favoreciendo la entrada de glucosa en el tejido muscular; también inhiben y retrasan la absorción de glucosa en intestino delgado. El mecanismo exacto por el cual inducen acidosis láctica es incierto. Se sabe que reducen la actividad de la piruvatodeshidrogenasa y el transporte mitocondrial de los agentes de óxido-reducción, aumentando así el metabolismo anaerobio, por lo que se acumulan los precursores para el ciclo de Krebs. La inhibición de la piruvato-deshidrogenasa da como resultado una disminución de la capacidad de metabolizar esos precursores en la ruta aerobia, aumentando el paso de piruvato a lactato, lo que conlleva a un aumento de la producción neta de ácido láctico. Además, la mayor absorción de glucosa en el intestino delgado podría elevar, teóricamente, los niveles de lactato en el sistema portal. La fenformina tiene una mayor propensión a causar acidosis láctica, debido a que su estructura química posee una cadena lateral muy lipofílica con gran afinidad para unirse a las membranas mitocondriales. Ello podría explicar su mayor capacidad para inhibir el metabolis-

TABLA I

EVOLUCIÓN ANALÍTICA

\begin{tabular}{lcccccc}
\hline FiO2 & $P H$ & PO2 & PCO2 & EAB & Sat O2 & HCO3 \\
\hline $21 \%$ & 7,21 & $61 \mathrm{mmHg}$ & $46 \mathrm{mmHg}$ & $-10 \mathrm{mmol} / \mathrm{L}$ & $86 \%$ & $18 \mathrm{mmol} / \mathrm{L}$ \\
$100 \%$ & 7,29 & $120 \mathrm{mmHg}$ & $45 \mathrm{mmHg}$ & $-5 \mathrm{mmol} / \mathrm{L}$ & $98 \%$ & $21 \mathrm{mmol} / \mathrm{L}$ \\
Gafas 41 & 7,44 & $126 \mathrm{mmHg}$ & $38 \mathrm{mmHg}$ & $3 \mathrm{mmol} / \mathrm{L}$ & $98 \%$ & $23 \mathrm{mmol} / \mathrm{L}$ \\
\hline
\end{tabular}

\begin{tabular}{lcccccc} 
Gluc. & Creat. & AST & ALT & CPK & Na & $K$ \\
\hline $659 \mathrm{mg} / \mathrm{dL}$ & $1,8 \mathrm{mg} / \mathrm{dL}$ & $120 \mathrm{U} / \mathrm{L}$ & $141 \mathrm{U} / \mathrm{L}$ & $28 \mathrm{U} / \mathrm{L}$ & $129 \mathrm{mmol} / \mathrm{L}$ & $9,6 \mathrm{mmol} / \mathrm{L}$ \\
$551 \mathrm{mg} / \mathrm{dL}$ & $1,9 \mathrm{mg} / \mathrm{dL}$ & $231 \mathrm{U} / \mathrm{L}$ & $305 \mathrm{U} / \mathrm{L}$ & $20 \mathrm{~L}$ & $131 \mathrm{mmol} / \mathrm{L}$ & $7,4 \mathrm{mmol} / \mathrm{L}$ \\
$146 \mathrm{mg} / \mathrm{dL}$ & $1,0 \mathrm{mg} / \mathrm{dL}$ & $226 \mathrm{U} / \mathrm{L}$ & $270 \mathrm{U} / \mathrm{L}$ & $35 \mathrm{U} / \mathrm{L}$ & $144 \mathrm{mmol} / \mathrm{L}$ & $3 \mathrm{mmol} / \mathrm{L}$ \\
$223 \mathrm{mg} / \mathrm{dL}$ & $0,9 \mathrm{mg} / \mathrm{dL}$ & $27 \mathrm{U} / \mathrm{L}$ & $96 \mathrm{U} / \mathrm{L}$ & $61 \mathrm{U} / \mathrm{L}$ & $149 \mathrm{mmol} / \mathrm{L}$ & $3,1 \mathrm{mmol} / \mathrm{L}$ \\
\hline
\end{tabular}


TABLA II

CLASIFICACIÓN ACIDOSIS LÁCTICA

Tipo A: Asociada a hipoperfusión y/o hipooxigenación tisular

Tipo B: En ausencia de hipoperfusión e hipooxigenación tisular

B1 0 asociada a diferentes enfermedades

B2 0 asociada a drogas y tóxicos

B3 o asociada a errores innatos del metabolismo

mo aerobio. Se ha comprobado que, cierto porcentaje de pacientes, presentaban un déficit hereditario para la hidroxilación de la fenformina, por lo que esta se acumulaba en plasma. Hay una serie de factores que favorecen el desarrollo de acidosis láctica. Cualquier situación que de lugar a hipoperfusión, con la consiguiente hipoxia tisular, favorece la conversión de piruvato a lactato (8). Patologías concurrentes como la insuficiencia hepática, EPOC, insuficiencia cardiaca, shock de cualquier etiología y la cardiopatía isquémica, favorecen el desarrollo de acidosis láctica en pacientes diabéticos en tratamiento con biguanidas. La sobredosificación también favorece el desarrollo de dicha acidosis $(9,10)$. La metformina se elimina esencialmente por secreción a través del túbulo renal, por lo que fármacos que interfieran disminuyendo la eliminación renal como, por ejemplo, la digoxina, procainamida, beta-bloqueantes, quinidina, contrastes iodados, ranitidina ó amiloride, aumentan los niveles plasmáticos favoreciendo la aparición de efectos secundarios (11).

La FDA (Food and Drug Administration) recogió, en 1996, 66 pacientes con acidosis láctica que estaban en tratamiento con metformina. De ellos se confirmaron 47 casos con elevación de ácido láctico en plasma, de los cuales 43 presentaban factores de riesgo para desarrollar acidosis láctica: $64 \%$ con enfermedad cardiaca, 18 con insuficiencia cardiaca congestiva, $28 \%$ con insuficiencia renal (incluidos dos pacientes en diálisis), $6 \%$ con antecedentes de EPOC, $17 \%$ mayores de 80 años y, finalmente, sólo 4 pacientes no tenían factores de riesgo cuando iniciaron tratamiento con metformina. Sobre datos basados en 1 millón de americanos en tratamiento con metformina, se han descrito, aproximadamente, 5 casos/100.000 que presentaban acidosis láctica plasmática. Stang y cols. en Saskatchewan (Canadá) aportaron 9 casos/100.000 (12). Misdin et al. describió tres casos, en pacientes que llevaban tratamiento con metformina, que fallecieron tras desarrollar acidosis láctica, estableciendo los criterios diagnósticos bioquímicos y clínicos (13).

La acidosis láctica secundaria a metformina se caracteriza, desde el punto vista clínico, por un inicio brusco acompañado de hiperventilación, dolor abdominal, somnolencia y coma, pudiendo llegar a PCR. Se describe un aumento de la incidencia en pacientes con patología asociada cardiaca, renal y hepática. El tratamiento se basa en el mantenimiento de las constantes vitales, tratamiento etiológico si se conoce, junto con la administración de bicarbonato, insulina ev, adecuada hidratación y aporte electrolítico. En ocasiones, en casos de mala evolución, puede llegar a ser necesario el empleo de hemodiálisis.

Podemos concluir que la asociación de acidosis lácticametformina es muy poco frecuente (Incidencia aproximada de 0,03-0,09 casos por 1.000 pacientes y año). La lactacidemia ocurre en pacientes diabéticos tipo 2 con función renal normal sin repercusión metabólica. El aumento del anión gap y la concurrencia de determinados datos de laboratorio, junto con la clínica, nos sirven para diagnosticar la acidosis láctica, siendo la determinación de lactacidemia la confirmación del diagnóstico. En nuestro caso falta la determinación de lactato en sangre, aunque la existencia de anión gap elevado y otros datos de laboratorio aportados, junto con la ausencia de otras posibles causas como la cetosis y la uremia, nos lleva a la conclusión que la causa más probable de la acidosis metabólica sea debida a la toma de Metformina a dosis altas favorecida por la concomitancia de factores predisponentes cardiovasculares (HTA mal controlada). Siendo la Metformina un fármaco de gran utilidad en el tratamiento de la diabetes mellitus en obesos con mal control metabólico, debemos tener en cuenta su empleo en estos pacientes que presentan factores concomitantes que favorezcan el desarrollo de acidosis metabólica así como aquellos fármacos que disminuyan la eliminación renal interfiriendo la secreción a dicho nivel. Así mismo debemos suspender y evitar el uso de Metformina en pacientes que han presentado episodio previo de acidosis metabólica por dicho fármaco.

\section{Bibliografía}

1. Lalau JD, Race JM. Metformin and lactic acidosis in diabetic humans. Diabetes Obes Metab 2000; 2:131-7.

2. Lalau JD, Race JM. Lactic acidosis in metformin therapy: searching for a link with metformin in reports of metformin-associated lactic acidosis. Diabetes Obes Metab 2001; 3:195-201.

3. Abbasi AA, Kasmikha R, Sotingeanu DG. Metformin-induced lacticacidemia in patients with type 2 diabetes mellitus. Endocr Pract 2000; 6: 442-6.

4. Jurovich MR, Wooldridge JD, Force RW. Metformin-associated nonketotic metabolic acidosis. Ann pharmacother 1997; 31: 53-5.

5. Lalau JD, Race JM. Lactic acidosis in metformin-treated patients. Prognostic value of arterial lactate levels and plasma metformin concentrations. Drug Saf 1999; 20: 3777-84.

6. Pepper GM, Schwartz M. Lactic acidosis associated with Glucophage use in a man with normal renal and hepatic function. Diabetes Care 1997; 20: 232-3

7. Jurovich MR, Wooldridge JD, Force RW. Metformin-associated nonke-

totic metabolic acidosis. Ann Pharmacother 1997; 31: 53-5.

8. Ellis AK, Iliescu EA. Metformin-associated lactic acidosis in a low risk patient. Can J Clin Pharmacol 2001; 8:104-6.

9. Al-Jebawi AF, Lassman MN, Abourizk NN. Lactic acidosis with therapeutic metformin blood level in a low-risk diabetic patient. Diabetes Care 1998; 21: 1364-5.

10. Klow NE, Draganov B, Os I. Metformin and contrast media-increased risk of lactic acidosis?. Tidsskr Nor Laegeforen 2001; 10; 121 : 1829.

11. Lalau JD, Lacroix C, Compagnon P, de Cagny B, Rigaud JP, et al. Role of metformin accumulation in metformin-associated lactic acidosis. Diabetes Care 1995; 18: 779-84.

12. Stang M, Wysowski DK, Butler-Jones D. Incidence of lactic acidosis in metformin users. Diabetes Care 1999; 22: 925-7.

13. Misbin RI, Green L, Stadel BV, Gueriguian JL, Gubbi A, Fleming GA. Lactic acidosis in patients with diabetes treated with metformin. N Engl J Med 1998; 338: 265-6. 